\title{
A FINITE VOLUME METHOD BASED ON THE CROUZEIX-RAVIART ELEMENT FOR ELLIPTIC PDE'S IN TWO DIMENSIONS
}

\author{
Panagiotis Chatzipantelidis \\ Department of Mathematics, University of Crete, 71409 Heraklion, Crete, Greece, and \\ Institute of Applied and Computational Mathematics, FO.R.T.H, 71110 Heraklion, Crete, Greece.
}

\begin{abstract}
A BSTRaCt. We introduce and analyse a finite volume method for the discretization of elliptic boundary value problems in $\mathbb{R}^{2}$. The method is based on nonuniform triangulations with piecewise linear nonconforming spaces. We prove optimal order error estimates in the $L^{2}$-norm and a mesh dependent $H^{1}$-norm.
\end{abstract}

\section{INTRODUCTION}

We consider an elliptic boundary value problem of the form: Seek a function $u: \Omega \subset \mathbb{R}^{2} \rightarrow \mathbb{R}$ such that

$$
\begin{aligned}
-\operatorname{div}(A \nabla u)+\sigma u & =f & & \text { in } \Omega, \\
u & =0 & & \text { on } \partial \Omega,
\end{aligned}
$$

with $\Omega$ a bounded, convex, polygonal domain in $\mathbb{R}^{2}, f \in L^{2}(\Omega), A=\left(a_{i j}\right)_{i, j=1}^{2}$ a given realvalued matrix function, $a_{i j} \in C^{1}(\bar{\Omega}), 1 \leq i, j \leq 2$, and $\sigma$ a smooth, nonnegative, real function, uniformly bounded in $\Omega$ by a constant $\bar{\sigma}$. We assume that the matrix $A$ is uniformly positive definite, i.e., there exists a positive constant $\alpha_{0}$ such that

$$
\xi^{T} A(x) \xi \geq \alpha_{0} \xi^{T} \xi, \quad \forall \xi \in \mathbb{R}^{2}, \forall x \in \bar{\Omega} .
$$

In this paper we will construct and analyse a finite volume method for the discretization of (1.1). The method is based on the "classical" finite volume method, where we approximate the solution of the problem by discretizing an integral formulation of the differential equation, on a finite partitioning of $\Omega$. We will seek an approximation of the solution of the problem in a space of nonconforming piecewise linear functions, the Crouzeix-Raviart finite element space, cf. [8].

Many researchers have analysed finite difference schemes constructed by a finite volume method. For example, Morton, Stynes and Süli, in [14], [15] and [17], treat cell-vertex schemes for convection-diffusion and hyperbolic problems on quadrilateral meshes. Also Süli, in [18], for Poisson's equation, Lazarov, Mishev and Vassilevski, in [12], for convection-diffusion problems and Ewing, Lazarov, Petrova and Vassilevski, in [10] and [20], for second order elliptic equations, analyse cell-centered schemes on quadrilateral and triangular grids.

1991 Mathematics Subject Classification. 65N30, 65N15.

E-mail: chatzipa@math.uch.gr

Supported in part by the Greek Secretariat for Research and Technology through the PENED program, \#1747. 
However, many researchers implement a finite volume method (called also box method or finite volume element method) for elliptic partial differential equations using finite element spaces, see for example Bank and Rose [1], Hackbusch [11], Cai, Mandel and McCormick [3] and [4], Schmidt [16]. In these works the approximations of the exact solution are sought in the standard space of continuous piecewise linear functions, on a given triangular or quadrilateral mesh. Based on this given mesh they construct a dual partition of $\Omega$, consisting of starshaped regions, in some cases overlapping, cf. [16], called boxes or volumes, having a one to one correspondence with the vertices of the mesh. The convergence of the resulting scheme, in a mesh dependent or discrete $H^{1}$-norm, is proved then by assuming various properties (depending on the simplicity of the elliptic equation (1.1)) for the initial mesh and the dual partition of boxes.

In the case of nonstructured triangular meshes, Bank and Rose, [1], analysed a finite volume method, which they called box method, for the Poisson equation and for an elliptic equation of the form (1.1), in the case $A(x)=a(x) I$, with $I$ the identity matrix. They consider a nonuniform triangulation of $\Omega$ satisfying the minimal angle condition, i.e. there exists a constant $\theta_{0}>0$ such that all angles of the triangles are bounded below by $\theta_{0}$. In order to construct the dual partition of $\Omega$ they choose a point, $z_{K}$, in each triangle $K$ and connect it with the middle of each side of $K$. The choice of $z_{K}$ is irrelevant for the analysis. However the extension of the convergence proof in the case of less simple elliptic equations is not straightforward. Cai, [3], analysed the same method with [1], for an elliptic equation of the form (1.1), with $\sigma=0$. He constructs the dual partition as in [1]. But the choice of the point $z_{K}$ inside each triangle is important for the analysis. So he considers either the circumcenter, orthocenter, incenter, or centroid of a triangle $K$. In order for the circumcenter and orthocenter to lie inside $K$, it is assumed that all angles of the triangles are bounded above by $\frac{\pi}{2}$. The convergence results of [3] are based on establishing the uniform coercivity of an auxiliary bilinear form. Therefore the convergence relies on giving sufficient conditions for this assumption to hold. In the case $A=I$ essentially no additional hypotheses are needed to establish this assumption, [3, Proposition 5.1]. In the case, however, of a general $A$ this assumption is verified (in the case where the volumes are constructed from circumcenters) provided that each triangle is either right or isosceles, [3, Theorem 5.1]. Recently in [5] using a different approach than [3], we proved optimal order $H^{1}$ and $L^{2}$ norm error estimates for (1.1), on triangulations satisfying only the minimal angle condition.

In our analysis, we will use as an approximation space nonconforming piecewise linear elements on a nonuniform triangulation of $\Omega$ satisfying the minimal angle condition. The boxes in the dual partition are again starshaped regions, but having a one to one correspondence with the sides of the triangulation. We construct the dual boxes by choosing a point $z_{K}$ inside every triangle $K$ and connecting it with the vertices of $K$. We prove optimal order convergence results in a mesh dependent $H^{1}$-norm and in the $L^{2}$-norm for the general problem (1.1), assuming only the minimal angle condition. The choice of $z_{K}$ is irrelevant for the derivation of the $H^{1}$-norm error estimate. However, in order to prove an optimal order $L^{2}$-norm error estimate, we assume that the given function $f$ is an element of $H^{1}(\Omega)$ and we choose $z_{K}$ to be the barycenter of $K$, cf. Theorems 3.2 and 4.2 .

A brief outline of the paper is the following. In section 2 we consider a family of nondegenerate triangulations of $\Omega$ with a corresponding dual partition of boxes and we introduce notation. In section 3 we consider a finite volume method for the approximation of the solution $u$ of the Poisson equation. The approximate solution $u_{B}$ is a linear polynomial on every triangle of the triangulation, not necessarily continuous on $\Omega$. Then in Theorems 3.1 and 3.2 we estimate the difference $u-u_{B}$ in a mesh dependent $H^{1}{ }^{-}$norm and in the $L^{2}{ }^{-}$norm, respectively. The analysis 

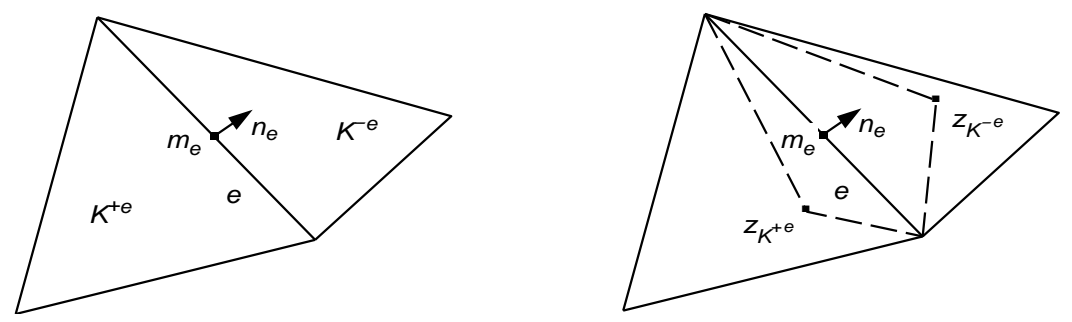

Figure 1. A sample region $V_{e}$. Left: The two triangles $K^{+e}$ and $K^{-e}$ with a common side $e$. Right: The box $b_{e}$ corresponding to side $e$ (with dotted lines).

in $\S 3$ is performed in order to introduce to the general case of equation (1.1). Indeed in $\S 4$ we consider a finite volume method for the approximation of the solution $u$ of problem (1.1) and in Theorems 4.1 and 4.2 we extend our convergence results to this case. In [13], Kossioris, Makridakis and Souganidis use a similar method to construct finite volume approximations to Hamilton-Jacobi equations.

\section{Notation}

Consider a bounded, convex polygonal domain $\Omega$ and a triangulation $T_{h}$ of $\Omega$. Let $h_{K}$ be the diameter of the triangle $K \in T_{h}$ and $h=\max _{K \in T_{h}} h_{K}$. We assume that the family $\left\{T_{h}\right\}_{0<h<1}$ of triangulations is nondegenerate, i.e. there exists a positive constant $\varrho$, independent of $h$, such that, for every $K \in T_{h}$,

$$
\varrho_{K} \geq \varrho h_{K}
$$

where by $\varrho_{K}$ we denote the diameter of the largest circle contained in $K \in T_{h}$, see, e.g., [2, p. 106]. This assumption is equivalent to the minimal angle condition, cf. [21].

Given a domain $K \subset \mathbb{R}^{2}$, denote by $L^{2}(K)$ the square integrable real functions over $K,(\cdot, \cdot)_{K}$ the inner product in $L^{2}(K),|\cdot|_{s, K}$ and $\|\cdot\|_{s, K}$ the seminorm and norm, respectively, of the Sobolev space $H^{s}(K), s \in \mathbb{N}$. Then, we introduce the norm $\|\cdot\|_{s, h},\|v\|_{s, h}=\left\{\sum_{K \in T_{h}}\|v\|_{s, K}^{2}\right\}^{1 / 2}$ and the seminorm $|\cdot|_{s, h},|v|_{s, h}=\left\{\sum_{K \in T_{h}}|v|_{s, K}^{2}\right\}^{1 / 2}, s \in \mathbb{N}$. Also with $|\cdot|$ denote either the area of a region in $\mathbb{R}^{2}$ or the length of a line segment, $\|\cdot\|_{\mathbb{R}^{2}}$ the Euclidean norm on $\mathbb{R}^{2}$ and also the subordinate matrix norm.

Given a triangle $K \in T_{h}$, let $E_{h}(K)$ be the set of the sides of $K$ and $E_{h}=\cup_{K \in T_{h}} E_{h}(K)$. Also, let $E_{h}^{\text {in }}$ be the set of the interior sides of the triangulation $T_{h}$, i.e. $e \in E_{h}^{\text {in }}$ if and only if $e \in E_{h}$ and $e$ is not part of $\partial \Omega$. With each side $e \in E_{h}^{\text {in }}$ we associate a region $V_{e}$, consisting of the two triangles of $T_{h}$ that have $e$ as a common side, let $T_{h}\left(V_{e}\right)$ be the set of the triangles of $V_{e}$ and denote by $m_{e}$ the middle point of a side $e \in E_{h}$ (see Figure 1).

We construct the dual partition of $T_{h}$ in the following way. Consider an interior point $z_{K}$ of $K \in T_{h}$ and connect it with line segments to the vertices of $K$. Thus we partition $K$ into three subtriangles, $K_{e}, e \in E_{h}(K)$. We denote this finer triangulation of $\Omega$ by $\widetilde{T_{h}}$ and for every $K \in T_{h}$, let $\widetilde{T_{h}}(K)$ be the set $\left\{\widetilde{K} \in \widetilde{T_{h}}: \widetilde{K}\right.$ subtriangle of $\left.K\right\}$. Now, with each side $e \in E_{h}^{\text {in }}$ we associate a region $b_{e}$ that consists of the two triangles of $\widetilde{T_{h}}$ that have $e$ as a common side and let $\mathcal{B}=\left\{b_{e}: e \in E_{h}^{\text {in }}\right\}$. Given also two triangles $K_{1}, K_{2} \in T_{h}$, with a common side $e \in E_{h}^{\text {in }}$, let 


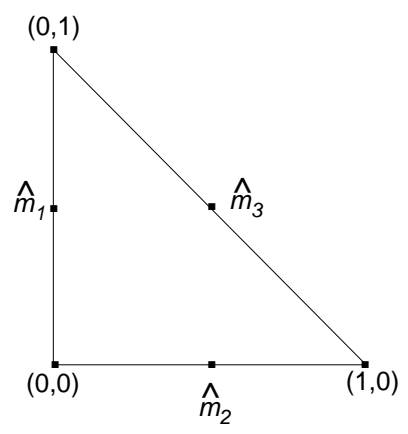

Figure 2. The reference triangle $\widehat{K}$.

$n_{e}$ be the normal vector of $K_{1}$ to $e$. Denote then $K_{1}$ by $K^{+e}$ and $K_{2}$ by $K^{-e}$ (see Figure 1 ). Also let $b_{e}^{+}, b_{e}^{-} \in \widetilde{T_{h}}$ be $b_{e}^{+}=K^{+e} \cap b_{e}$ and $b_{e}^{-}=K^{-e} \cap b_{e}$.

Next, let $S_{h}$ be the nonconforming, piecewise linear finite element space consisting of functions which reduce to linear polynomials in any $K \in T_{h}$ and are continuous at the points $m_{e}, e \in E_{h}^{\text {in }}$, cf. [8]. Also denote by $S_{h}^{0}$ the subspace $\left\{v \in S_{h}: v\left(m_{e}\right)=0, e \in E_{h} \backslash E_{h}^{\mathrm{in}}\right\}$. Note that $S_{h}$ is not a subspace of $H^{1}(\Omega)$. For any $K \in T_{h}$, let $v_{K}$ denote the restriction of $v \in S_{h}$ in $K$. The functions $\varphi_{e}, e \in E_{h}^{\text {in }}$, such that

$$
\operatorname{supp} \varphi_{e}=V_{e}, \quad \varphi_{e}\left(m_{e}\right)=1 \quad \text { and } \varphi_{e}\left(m_{\ell}\right)=0, \quad \forall \ell \in E_{h} \backslash\{e\},
$$

form a basis of $S_{h}^{0}$ (see, e.g., [19, p. 113]).

Consider now a reference triangle $\widehat{K}$ with vertices $(0,0),(1,0),(0,1)$. Let $\widehat{e}_{1}, \widehat{e}_{2}$ and $\widehat{e}_{3}$, be the sides of $\widehat{K}$, starting from the side opposite to the vertex $(1,0)$ and continuing anticlockwise. Also denote by $\widehat{m}_{i}$ the middle point of side $\widehat{e}_{i}, i=1,2,3$, and $\widehat{\varphi}_{i}, i=1,2,3$, the first degree polynomial in $\widehat{K}$ such that $\widehat{\varphi}_{i}\left(\widehat{m}_{j}\right)=\delta_{i j}, i, j=1,2,3$, (see Figure 2 ).

Next, let $\widehat{S}(\widehat{K}):=\operatorname{span}\left\{\widehat{\varphi}_{1}, \widehat{\varphi}_{2}, \widehat{\varphi}_{3}\right\}$, and for $e \in E_{h}(K)$ denote by $A_{K, e}$ a linear transformation of $\widehat{K}$ to $K \in T_{h}$, such that $m_{e}=A_{K, e} \widehat{m}_{3}$. Also for $\widehat{x} \in \widehat{K}$ let $A_{K, e} \widehat{x}=a_{K, e} \widehat{x}+b_{K, e}$, with $a_{K, e} \in G L\left(\mathbb{R}^{2}\right), b_{K, e} \in \mathbb{R}^{2}$ and for a function $v \in S_{h}^{0}$ denote by $\widehat{v}_{K, e}$ the function of $\widehat{S}(\widehat{K})$ defined by $\widehat{v}_{K, e}(\widehat{x})=v_{K}\left(A_{K, e} \widehat{x}\right)$.

\section{The Poisson equation}

As a motivation of the analysis of a finite volume method for a general elliptic operator $A$, we study in this section the finite volume method for the simplest elliptic operator, $-\Delta$. Thus, we consider the problem: Seek a function $u: \Omega \subset \mathbb{R}^{2} \rightarrow \mathbb{R}$ such that

$$
-\Delta u=f \quad \text { in } \Omega \quad \text { and } \quad u=0 \quad \text { on } \partial \Omega
$$

with $\Omega$ a bounded, convex, polygonal domain in $\mathbb{R}^{2}$ and $f \in L^{2}(\Omega)$.

We formulate the finite volume method for problem (3.1) in the following way. Consider a side $e \in E_{h}^{\text {in }}$ and the associated box, $b_{e}$. Then integrating (3.1) over $b_{e}$, and using Green's formula, we get

$$
-\int_{\partial b_{e}} \frac{\partial u}{\partial n} d s=\int_{b_{e}} f d x
$$


Having relation (3.2) in mind we formulate the finite volume method for (3.1) as: Find $u_{B} \in S_{h}^{0}$ such that

$$
-\int_{\partial b_{e}} \frac{\partial u_{B}}{\partial n} d s=\int_{b_{e}} f d x, \quad \forall e \in E_{h}^{\mathrm{in}} .
$$

In order to show existence and uniqueness of the solution of (3.3), we will first prove some lemmas that will be used in the analysis of problem (3.1) and also for the more general problem (1.1). Consider now a triangle $K \in T_{h}$ and a side $e \in E_{h}^{\text {in }} \cap E_{h}(K)$. Let $n_{e}$ be the external normal vector of $K$ to $e$. According to section 2 we denote by $K^{+e}$ triangle $K$ and by $K^{-e}$ the other triangle of $T_{h}$, having $e$ as a common side with $K$. Let now,

$$
\left[\frac{\partial \chi}{\partial n_{e}}\right]=\frac{\partial \chi_{K^{+e}}}{\partial n_{e}}-\frac{\partial \chi_{K^{-e}}}{\partial n_{e}}, \quad \forall \chi \in S_{h}^{0} .
$$

Lemma 3.1: Let $K \in T_{h}, e \in E_{h}^{\text {in }} \cap E_{h}(K)$ and $v \in S_{h}^{0}$. Then,

$$
\int_{\partial b_{e}} \frac{\partial v}{\partial n} d s=-\int_{e}\left[\frac{\partial v}{\partial n_{e}}\right] d s
$$

with $n_{e}$ the normal vector of $K$ to $e$.

Proof: Let $v \in S_{h}^{0}, K \in T_{h}, e \in E_{h}^{\text {in }} \cap E_{h}(K)$ and $n_{e}$ the external normal vector of $K$ to $e$. Using Green's formula we have

$$
\int_{\partial V_{e}} \frac{\partial v}{\partial n} d s=\int_{\partial K^{+e}} \frac{\partial v}{\partial n} d s+\int_{\partial K^{-e}} \frac{\partial v}{\partial n} d s-\int_{e}\left[\frac{\partial v}{\partial n_{e}}\right] d s .
$$

Combining now the relation above with the fact that $\Delta v=0$ over any $K \in T_{h}$, as well as over $K^{+e} \backslash b_{e}$ and $K^{-e} \backslash b_{e}$, for any $e \in E_{h}^{\text {in }}$, we obtain (3.5).

In view of Lemma 3.1 we can rewrite (3.3) as: Find $u_{B} \in S_{h}^{0}$ such that

$$
\int_{e}\left[\frac{\partial u_{B}}{\partial n_{e}}\right] d s=\int_{b_{e}} f d x, \quad \forall e \in E_{h}^{\mathrm{in}}
$$

where $n_{e}$ is the external normal vector of $K^{+e}$ to $e$, cf. (3.4) and Figure 1. We now define the bilinear forms $a, \bar{a}:\left(H^{1}(\Omega)+S_{h}\right) \times\left(H^{1}(\Omega)+S_{h}\right) \rightarrow \mathbb{R}$ by

$$
a(v, w)=\sum_{K \in T_{h}}(\nabla v, \nabla w)_{K} \quad \text { and } \quad \bar{a}(v, w)=-\sum_{e \in E_{h}^{\text {in }}} w\left(m_{e}\right) \int_{\partial b_{e}} \frac{\partial v}{\partial n} d s .
$$

Then, we can rewrite (3.3) as

$$
\bar{a}\left(u_{B}, \chi\right)=\sum_{e \in E_{h}^{\text {in }}} \chi\left(m_{e}\right) \int_{b_{e}} f d x .
$$

Remark 3.1: In view of (3.8), the finite volume method (3.3) can be viewed as a Petrov-Galerkin finite element method, where $S_{h}^{0}$ is the approximation space and the piecewise constants on $\mathcal{B}$ are the test functions. 
Lemma 3.2: Let $\chi, \psi \in S_{h}^{0}$. Then,

$$
\sum_{e \in E_{h}^{\text {in }}} \psi\left(m_{e}\right) \int_{e}\left[\frac{\partial \chi}{\partial n_{e}}\right] d s=a(\chi, \psi),
$$

with $n_{e}$ as in (3.4).

Proof: Since $\psi \in S_{h}^{0}$, we can express it as a linear combination of the basis elements of $S_{h}^{0}$, $\psi=\sum_{e \in E_{h}^{\text {in }}} \psi\left(m_{e}\right) \varphi_{e}$, and thus, in view of $(2.2)$,

$$
a(\chi, \psi)=\sum_{K \in T_{h}}(\nabla \chi, \nabla \psi)_{K}=\sum_{e \in E_{h}^{\text {in }}} \psi\left(m_{e}\right) \sum_{K \in T_{h}\left(V_{e}\right)}\left(\nabla \chi, \nabla \varphi_{e}\right)_{K}
$$

For $e \in E_{h}^{\text {in }}$ and $\chi \in S_{h}^{0}$, we have

$$
\sum_{K \in T_{h}\left(V_{e}\right)}\left(\nabla \chi, \nabla \varphi_{e}\right)_{K}=\sum_{K \in T_{h}\left(V_{e}\right)} \int_{\partial K} \frac{\partial \chi}{\partial n} \varphi_{e} d s=\int_{\partial V_{e}} \frac{\partial \chi}{\partial n} \varphi_{e} d s+\left[\frac{\partial \chi}{\partial n_{e}}\right] \int_{e} \varphi_{e} d s
$$

Since $\varphi_{e}$ is a linear polynomial on every side of $K \in T_{h}\left(V_{e}\right)$, we get

$$
\int_{\ell} \varphi_{e} d s=|\ell| \varphi_{e}\left(m_{\ell}\right), \quad \forall \ell \in \cup_{K \in T_{h}\left(V_{e}\right)} E_{h}(K)
$$

$|\ell|$ denoting the length of the side $\ell$. Therefore, combining (3.10)-(3.12) and (2.2) we easily obtain (3.9).

Existence: It follows easily in view of Lemmas 3.1 and 3.2.

Remark 3.2: The Galerkin/finite element method, using $S_{h}^{0}$ as the approximation space is: Find $u_{G} \in S_{h}^{0}$ such that

$$
a\left(u_{G}, \chi\right)=(f, \chi)_{\Omega}, \quad \forall \chi \in S_{h}^{0}
$$

Thus, in view of Lemmas 3.1 and 3.2 we notice that the finite volume method (3.8) and the Galerkin finite element method (3.13) reduce both to linear systems with the same matrix; they only differ in the right-hand side terms. Motivated by this observation it is possible to take a different approach in th error analysis that will be closer to the analysis of the finite element case, cf. [5]. Note that a similar relation is not true for the general case of equation (1.1) considered in $\S 4$.

Lemma 3.3: There exist positive constants $C(\varrho)$ and $C_{\star}(\varrho)$, independent of $h$, such that, for every $v \in H^{1}(K)$ and $K \in T_{h}$,

$$
\begin{gathered}
C|v|_{1, K}^{2} \leq\left|\widehat{v}_{K, e}\right|_{1, \widehat{K}}^{2} \leq C^{-1}|v|_{1, K}^{2}, \\
\text { and } \quad C_{\star}\|v\|_{0, K}^{2} \leq h_{K}^{2}\left\|\widehat{v}_{K, e}\right\|_{0, \widehat{K}}^{2} \leq C_{\star}^{-1}\|v\|_{0, K}^{2},
\end{gathered}
$$

where $e \in E_{h}(K)$ and $\varrho$ the constant in (2.1). 
Proof: Let $K \in T_{h}, e \in E_{h}(K)$ and $A_{K, e}$ be a linear transformation of $\widehat{K}$ to $K$ with $m_{e}=$ $A_{K, e} \widehat{m}_{3}$. According to [6, Theorems 15.1 and 15.2], and the notation introduced in $\S 2$, there exists a constant $C$ independent of $K$ such that, for all $v \in H^{\ell}(K), \ell \in \mathbb{N}$,

$$
\begin{gathered}
|v|_{\ell, K} \leq\left|\operatorname{det} a_{K, e}\right|^{1 / 2}\left\|a_{K, e}^{-1}\right\|_{\mathbb{R}^{2}}^{\ell}\left|\widehat{v}_{K, e}\right|_{\ell, \widehat{K}}, \quad\left|\widehat{v}_{K, e}\right|_{\ell, \widehat{K}} \leq\left|\operatorname{det} a_{K, e}\right|^{-1 / 2}\left\|a_{K, e}\right\|_{\mathbb{R}^{2}}^{\ell}|v|_{\ell, K}, \\
\left\|a_{K, e}^{-1}\right\|_{\mathbb{R}^{2}} \leq C \varrho_{K}^{-1}, \quad\left\|a_{K, e}\right\|_{\mathbb{R}^{2}} \leq C h_{K} .
\end{gathered}
$$

Obviously, $|K|=\frac{1}{2}\left|\operatorname{det} a_{K, e}\right|$ and $|K| \leq C h_{K}^{2}$, where again by $|\cdot|$ we denote the area of a region in $\mathbb{R}^{2}$. Thus in view of (3.16) and (2.1), we easily obtain

$$
|v|_{1, K}^{2} \leq C \varrho^{-2}\left|\widehat{v}_{K, e}\right|_{1, \widehat{K}}^{2} \quad \text { and } \quad\|v\|_{0, K}^{2} \leq C h_{K}^{2}\left\|\widehat{v}_{K, e}\right\|_{0, \widehat{K}}^{2} .
$$

Hence, the first inequalities of (3.14) and (3.15) hold. Using now the fact that $|K| \geq\left|B_{K}\right| \geq$ $C \varrho^{2} h_{K}^{2}$, with $B_{K}$ the largest circle contained in $K$, and similar arguments as before we obtain the second inequalities of (3.14) and (3.15).

Next, by simple calculations one can prove the following three lemmas.

Lemma 3.4: Let $v \in \widehat{S}(\widehat{K})$; then

$$
|v|_{1, \widehat{K}}^{2}=2\left\{\left(v\left(\widehat{m}_{3}\right)-v\left(\widehat{m}_{1}\right)\right)^{2}+\left(v\left(\widehat{m}_{3}\right)-v\left(\widehat{m}_{2}\right)\right)^{2}\right\} .
$$

Lemma 3.5: There exists a constant $C$ such that, for $v \in S_{h}^{0}$,

$$
C|v|_{1, h}^{2} \leq \sum_{K \in T_{h}} \sum_{e, \ell \in E_{h}(K)}\left(v\left(m_{\ell}\right)-v\left(m_{e}\right)\right)^{2} \leq C^{-1}|v|_{1, h}^{2}
$$

Lemma 3.6: Consider a triangulation $T_{h}$ of a convex, bounded, polygonal domain $\Omega$, satisfying (2.1). Then there exists a constant $C(\varrho)$, independent of $h$ and $\varepsilon$, such that, for every $v \in L^{2}(\Omega)$ such that $\left.v\right|_{K} \in H^{1}(K)$ for every $K \in T_{h}$

$$
\int_{\partial K} v^{2} d s \leq C\left(\varepsilon^{-1 / 2} h_{K}^{-1}\|v\|_{0, K}^{2}+\varepsilon^{1 / 2} h_{K}|v|_{1, K}^{2}\right), \quad \forall K \in T_{h},
$$

with $\varepsilon \in(0,1)$.

Proof: According to [9, Theorem 1.5.1.10], there exists a constant $C(\widehat{K})$ such that

$$
\int_{\partial \widehat{K}} w^{2} d \widehat{s} \leq C\left(\varepsilon^{-1 / 2}\|w\|_{0, \widehat{K}}^{2}+\varepsilon^{1 / 2}|w|_{1, \widehat{K}}^{2}\right), \quad \forall w \in H^{1}(\widehat{K}) .
$$

Consider now a triangle $K \in T_{h}$. Using then (3.21) and repeating similar arguments as in Lemma 3.3 for a function $v \in H^{1}(K)$, we see that there exists a constant $C(\varrho)$, independent of $K \in T_{h}$, such that

$$
\int_{\partial K} v^{2} d s \leq C\left(\varepsilon^{-1 / 2} h_{K}^{-1}\|v\|_{0, K}^{2}+\varepsilon^{1 / 2} h_{K}|v|_{1, K}^{2}\right) .
$$

Hence, we can easily see that (3.20) holds.

In the sequel we state Proposition 4.13 of [19] as Lemma 3.7. 
Lemma 3.7 (Discrete Poincaré Inequality): There exists a positive constant $C$ such that

$$
\|v\|_{0, \Omega} \leq C|v|_{1, h}, \quad \forall v \in S_{h}^{0} .
$$

Next, we will estimate the difference $u-u_{B}$, in a mesh dependent $H^{1}$-norm.

Theorem 3.1: Let $u$ be the solution of (3.1) and $u_{B}$ the solution of (3.3). There exists a constant $C$, independent of $h$, such that

$$
\left\|u-u_{B}\right\|_{1, h} \leq C\left(\sum_{K \in T_{h}} h_{K}^{2}|u|_{2, K}^{2}\right)^{1 / 2} .
$$

Proof: Combining (3.2) and (3.3), we have

$$
\bar{a}\left(u-u_{B}, v\right)=0, \quad \forall v \in S_{h}^{0}
$$

Using now Lemmas 3.1, 3.2 and (3.24), we have for any $\chi \in S_{h}^{0}$

$$
\left\|\nabla\left(u_{B}-\chi\right)\right\|_{0, h} \leq \sup _{\substack{v \in S_{h}^{0} \\ v \neq 0}} \frac{a\left(u_{B}-\chi, v\right)}{|v|_{1, h}}=\sup _{\substack{v \in S_{h}^{0} \\ v \neq 0}} \frac{\bar{a}\left(u_{B}-\chi, v\right)}{|v|_{1, h}}=\sup _{\substack{v \in S_{h}^{0} \\ v \neq 0}} \frac{\bar{a}(u-\chi, v)}{|v|_{1, h}}
$$

Next, applying the Cauchy-Schwarz inequality, Lemma 3.5, and Green's formula, and using the fact that $\chi$ is a linear polynomial in $K \in T_{h}$, we have the following estimation for all $v, \chi \in S_{h}^{0}$

$$
\begin{aligned}
& |\bar{a}(u-\chi, v)|^{2}=\left|\sum_{e \in E_{h}^{\text {in }}} v\left(m_{e}\right) \int_{\partial b_{e}} \frac{\partial(u-\chi)}{\partial n} d s\right|^{2} \\
& =\frac{1}{2}\left|\sum_{K \in T_{h}} \sum_{e, \ell \in E_{h}(K)}\left(v\left(m_{e}\right)-v\left(m_{\ell}\right)\right) \int_{\partial b_{e} \cap \partial b_{\ell}} \frac{\partial(u-\chi)}{\partial n_{e}} d s\right|^{2} \\
& \leq C|v|_{1, h}^{2} \sum_{K \in T_{h}}\left(h_{K} \int_{\partial K}\left|\frac{\partial(u-\chi)}{\partial n}\right|^{2} d s+h_{K}^{2}|u|_{2, K}^{2}\right),
\end{aligned}
$$

with $n_{e}$ denoting the external normal vector of $b_{e} \in \mathcal{B}$. Consider next a triangle $K \in T_{h}$. According to Lemma 3.6, for $\varepsilon=1 / 2$ and $u, \chi$ as above, we obtain

$$
h_{K} \int_{\partial K}\left|\frac{\partial(u-\chi)}{\partial n}\right|^{2} d s \leq C\left(h_{K}^{2}|u|_{2, K}^{2}+\|u-\chi\|_{1, K}^{2}\right) \text {, }
$$

Next, consider the interpolation operator $I: H^{2}(\Omega) \rightarrow S_{h}, I u\left(m_{e}\right)=u\left(m_{e}\right)$, for all $e \in E_{h}$. Choosing now $\chi=I u$ in (3.25)-(3.27) and using Lemma 3.7 we get

$$
\left\|u-u_{B}\right\|_{1, h} \leq C\left(\|u-I u\|_{1, h}^{2}+\sum_{K \in T_{h}} h_{K}^{2}|u|_{2, K}^{2}\right)^{1 / 2} .
$$


Finally, in view of a standard approximation property of $I$, cf., e.g., [7, Theorem 5],

$$
\|u-I u\|_{1, h}^{2} \leq C \sum_{K \in T_{h}} h_{K}^{2}|u|_{2, K}^{2},
$$

(3.28) gives the desired estimate (3.23).

Remark 3.3: It is well known that the Galerkin/finite element method, (3.13), satisfies a similar $H^{1}$ norm error estimate, cf., e.g., [2, Chapter 8.3].

Denote now by $\mathbb{P}_{\mu}$ the set of polynomials of degree at most $\mu \in \mathbb{N}$, in $\mathbb{R}^{2}$, and by $\mathcal{M}_{G}^{\mu}$ the orthogonal projection operator from $L^{2}(G)$ onto $\mathbb{P}_{\mu}, G \subset \mathbb{R}^{2}$, defined by

$$
\int_{G} w \mathcal{M}_{G}^{\mu} v d s=\int_{G} w v d s, \quad \forall w \in \mathbb{P}_{\mu}
$$

Then, according to $\left[8\right.$, Lemma 3], we have for $K \in T_{h}$

$$
\left|\int_{e} \varphi\left(v-\mathcal{M}_{e}^{0} v\right) d s\right| \leq C h_{K}|\varphi|_{1, K}|v|_{1, K}, \quad \forall e \in E_{h}(K), \forall \varphi, v \in H^{1}(K) .
$$

In the sequel we shall use the fact that if $v, w \in H^{2}(V)$ with $V=K_{1} \cup K_{2}, K_{1}, K_{2} \in T_{h}$ and $\partial K_{1} \cap \partial K_{2}=e \in E_{h}$, then

$$
\int_{e} \nabla v \cdot n_{1} w d s+\int_{e} \nabla v \cdot n_{2} w d s=0
$$

with $n_{i}$ the external normal vector of $K_{i}$ to $e, i=1,2$. Next, we will estimate the difference $u-u_{B}$ in the $L^{2}$-norm.

Theorem 3.2: Let $u$ be the solution of (3.1) and $u_{B}$ the solution of (3.3). Assume that $f \in H^{1}(\Omega)$ and that the interior point $z_{K}$ is the barycenter of $K$, for every $K \in T_{h}$. Then, there exists a constant $C$, independent of $h$, such that

$$
\left\|u-u_{B}\right\|_{0, \Omega} \leq C h^{2}\left(\|u\|_{2, \Omega}+\|f\|_{1, \Omega}\right) .
$$

Proof: We consider the following auxiliary problem: Seek $\varphi \in H^{2}(\Omega)$ such that

$$
-\Delta \varphi=u-u_{B} \quad \text { in } \Omega \quad \text { and } \quad \varphi=0 \quad \text { on } \partial \Omega .
$$

We will use the well-known estimate, cf., e.g., [9, Chapter 4],

$$
\|\varphi\|_{2, \Omega} \leq C\left\|u-u_{B}\right\|_{0, \Omega} .
$$

Using (3.34) and Green's formula, we easily obtain

$$
\left\|u-u_{B}\right\|_{0, \Omega}^{2}=a\left(u-u_{B}, \varphi-v\right)+a\left(u-u_{B}, v\right)-\sum_{K \in T_{h}} \int_{\partial K}\left(u-u_{B}\right) \frac{\partial \varphi}{\partial n} d s, \quad \forall v \in S_{h}^{0}
$$

In the sequel we will estimate the three terms on the right-hand side of (3.36). Obviously that

$$
a\left(u-u_{B}, \varphi-v\right) \leq\left\|u-u_{B}\right\|_{1, h}\|\varphi-v\|_{1, h}, \quad \forall v \in S_{h}^{0} .
$$


In view of Lemmas 3.2 and $3.1,(3.1)$ and $(3.8)$, we rewrite the second term of the right-hand side of (3.36) as

$$
\begin{gathered}
a\left(u-u_{B}, v\right)=\sum_{K \in T_{h}} \int_{\partial K} \nabla u \cdot n v d s+\sum_{K \in T_{h}} \int_{K} f v d x-\sum_{e \in E_{h}^{\text {in }}} v\left(m_{e}\right) \int_{b_{e}} f d x \\
=\sum_{K \in T_{h}} \int_{\partial K} \nabla u \cdot n v d s+\sum_{K \in T_{h}} \int_{K} f(v-Q(v)) d x, \quad \forall v \in S_{h}^{0}
\end{gathered}
$$

with

$$
\left.Q(v)\right|_{K}=\sum_{e \in E_{h}(K)} v\left(m_{e}\right) g_{K_{e}}, \quad K \in T_{h}
$$

where $K_{e} \in \widetilde{T_{h}}(K)$ and $g_{S}$ denotes the characteristic function of a set $S \subset \mathbb{R}^{2}$. Now, we will estimate the first sum in the last relation of (3.38), compare to [8, (3.41)]. Using the definition of the projection $\mathcal{M}_{e}^{0},(3.30),(3.32)$, the fact that $v_{K^{+e}}\left(m_{e}\right)=v_{K^{-e}}\left(m_{e}\right)$, for every $e \in E_{h}^{\text {in }}$, $v \in S_{h}^{0}$ and $u \in H^{2}(\Omega)$, we get

$$
\begin{aligned}
& \sum_{K \in T_{h}} \sum_{e \in E_{h}(K)} \int_{e} \mathcal{M}_{e}^{0}(\nabla u \cdot n) v d s=\sum_{e \in E_{h}^{\text {in }}}\left(\int_{e} \mathcal{M}_{e}^{0}\left(\nabla u \cdot n_{e}\right) v_{K^{+e}} d s\right. \\
& \left.\quad-\int_{e} \mathcal{M}_{e}^{0}\left(\nabla u \cdot n_{e}\right) v_{K^{-e}} d s\right)+\sum_{e \in E_{h} \backslash E_{h}^{\text {in }}} \int_{e} \mathcal{M}_{e}^{0}\left(\nabla u \cdot n_{e}\right) v d s \\
& =\sum_{e \in E_{h}^{\text {in }}} \mathcal{M}_{e}^{0}\left(\nabla u \cdot n_{e}\right)\left(v_{K^{+e}}-v_{K^{-e}}\right)\left(m_{e}\right)+\sum_{e \in E_{h} \backslash E_{h}^{\text {in }}} \mathcal{M}_{e}^{0}\left(\nabla u \cdot n_{e}\right) v\left(m_{e}\right)=0, \forall v \in S_{h}^{0},
\end{aligned}
$$

Thus, in view of (3.40) we get

$$
\begin{aligned}
& \sum_{K \in T_{h}} \int_{\partial K} \nabla u \cdot n v d s=\sum_{K \in T_{h}} \sum_{e \in E_{h}(K)} \int_{e}\left(\nabla u \cdot n-\mathcal{M}_{e}^{0} \nabla u \cdot n\right) v d s, \quad \forall v \in S_{h}^{0} \\
& \text { and } \quad \sum_{K \in T_{h}} \sum_{e \in E_{h}(K)} \int_{e} \varphi \mathcal{M}_{e}^{0}(\nabla u \cdot n) d s=\sum_{K \in T_{h}} \sum_{e \in E_{h}(K)} \int_{e} \nabla u \cdot n \varphi d s=0 .
\end{aligned}
$$

Therefore, in view of (3.41) and (3.31) we obtain

$$
\begin{aligned}
\left|\sum_{K \in T_{h}} \int_{\partial K} \nabla u \cdot n v d s\right| & =\left|\sum_{K \in T_{h}} \sum_{e \in E_{h}(K)} \int_{e}\left(\nabla u \cdot n-\mathcal{M}_{e}^{0} \nabla u \cdot n\right)(v-\varphi) d s\right| \\
& \leq C h|u|_{2, \Omega}\|\varphi-v\|_{1, h}, \quad \forall v \in S_{h}^{0} .
\end{aligned}
$$

Next we estimate the last term of (3.38). To this end, let $C_{K}(f)=\int_{B} f \omega_{K} d x$, where $B$ is a ball in $\mathbb{R}^{2}$, satisfying $B \subset \subset K$ and $\omega_{K}$ a cut-off function supported in $\bar{B}$, cf. [2, Def. 4.1.3]. Then, according to the Bramble-Hilbert lemma, in the form given in [2, Lemma 4.3.8], we obtain in view of $(2.1)$

$$
\left\|f-C_{K}(f)\right\|_{0, K} \leq C(\varrho) h_{K}|f|_{1, K}, \quad \forall f \in H^{1}(K) .
$$


Since $C_{K}(f)$ is a constant over $K$, we have

$$
\int_{K} f(v-Q(v)) d x=\int_{K}\left(f-C_{K}(f)\right)(v-Q(v)) d x+C_{K}(f) \int_{K}(v-Q(v)) d x, \quad \forall v \in S_{h}^{0}
$$

Since, $z_{K}$ is the barycenter of $K \in T_{h}$, it is easily seen that

$$
\int_{K}(v-Q(v)) d x=\int_{K} v d x-\frac{|K|}{3} \sum_{e \in E_{h}(K)} v\left(m_{e}\right)=0, \quad \forall v \in \mathbb{P}_{2} .
$$

Also, we easily obtain

$$
\|v-Q(v)\|_{0, K} \leq C h_{K}|v|_{1, K}, \quad \forall v \in \mathbb{P}_{2} .
$$

Thus, using (3.43)-(3.46) and the fact that $f \in H^{1}(K)$, we get

$$
\left|\int_{K} f(v-Q(v)) d x\right| \leq C h_{K}^{2}|f|_{1, K}|v|_{1, K} \leq C h_{K}^{2}|f|_{1, K}\left(|\varphi-v|_{1, K}+|\varphi|_{1, K}\right), \quad \forall v \in S_{h}^{0}
$$

Therefore, combining (3.38), (3.42) and (3.47), we obtain

$$
\left|a\left(u-u_{B}, v\right)\right| \leq C h\left(\|u\|_{2, \Omega}+\|f\|_{1, \Omega}\right)\left(\|\varphi-v\|_{1, h}+h\|\varphi\|_{1, \Omega}\right), \quad \forall v \in S_{h}^{0}
$$

Finally, we estimate the last term in (3.36). Since $u-u_{B} \in L^{2}(\Omega), \varphi \in H^{2}(\Omega)$. Therefore, using similar arguments as in (3.40), in view of (3.30)-(3.32), the fact that $u_{B} \in S_{h}^{0}$ and $u \in H^{2}(\Omega)$, we obtain

$$
\begin{aligned}
\left|\sum_{K \in T_{h}} \int_{\partial K} \nabla \varphi \cdot n\left(u-u_{B}\right) d s\right| & =\left|\sum_{K \in T_{h}} \sum_{e \in E_{h}(K)} \int_{e}\left(\nabla \varphi \cdot n-\mathcal{M}_{e}^{0} \nabla \varphi \cdot n\right)\left(u-u_{B}\right) d s\right| \\
& \leq C h|\varphi|_{2, \Omega}\left\|u-u_{B}\right\|_{1, h} .
\end{aligned}
$$

Therefore, choosing $v=I \varphi$ in (3.36) and using (3.37), (3.48), (3.49), (3.35), (3.23), and (3.29), we have

$$
\begin{aligned}
\left\|u-u_{B}\right\|_{0, \Omega}^{2} & \leq\left(\left\|u-u_{B}\right\|_{1, h}+C h\left(\|u\|_{2, \Omega}+\|f\|_{1, \Omega}\right)\right)\left(\|\varphi-I \varphi\|_{1, h}+h\|\varphi\|_{2, \Omega}\right) \\
& \leq C h^{2}\left(\|u\|_{2, \Omega}+\|f\|_{1, \Omega}\right)\left\|u-u_{B}\right\|_{0, \Omega} .
\end{aligned}
$$

Remark 3.4: In the proof of Theorem 3.2, the hypothesis $f \in H^{1}(\Omega)$, is only used in (3.43) in the estimate of the last term of (3.38). It is known, cf. [8], that the corresponding $L^{2}$ norm error estimate for the finite element method (3.13) holds without the term $\|f\|_{1, \Omega}$ in the right-hand side. 


\section{A general elliptic EQUATion}

In this section we will construct and analyse a finite volume method for problem (1.1). Integrating (1.1) over $b_{e}$ and applying Green's formula, we obtain

$$
-\int_{\partial b_{e}}(A \nabla u) \cdot n d s+\int_{b_{e}} \sigma u d x=\int_{b_{e}} f d x, \quad \forall e \in E_{h}^{\mathrm{in}} .
$$

We formulate the finite volume method for this problem as: Find $u_{B} \in S_{h}^{0}$ such that

$$
-\int_{\partial b_{e}}\left(A \nabla u_{B}\right) \cdot n d s+u_{B}\left(m_{e}\right) \int_{b_{e}} \sigma d x=\int_{b_{e}} f d x, \quad \forall e \in E_{h}^{\text {in }} .
$$

As in [1], in order to discretize the reaction term, $\sigma u$, we do not generalize the Galerkin formulation. Instead, we choose the "classical" finite volume approach; we discretize it using a diagonal matrix, while the Galerkin approach would have resulted in a nondiagonal matrix. We redefine now the bilinear forms $a, \bar{a}:\left(H^{1}(\Omega)+S_{h}\right) \times\left(H^{1}(\Omega)+S_{h}\right) \rightarrow \mathbb{R}$ by

$$
a(v, w)=\sum_{K \in T_{h}} \int_{K}(A \nabla v) \cdot \nabla w d x \quad \text { and } \quad \bar{a}(v, w)=-\sum_{e \in E_{h}^{\text {in }}} w\left(m_{e}\right) \int_{\partial b_{e}}(A \nabla v) \cdot n d s .
$$

Then, we can rewrite (4.2) as

$$
\bar{a}\left(u_{B}, v\right)+\sum_{e \in E_{h}^{\text {in }}} v\left(m_{e}\right) u_{B}\left(m_{e}\right) \int_{b_{e}} \sigma d x=\sum_{e \in E_{h}^{\text {in }}} v\left(m_{e}\right) \int_{b_{e}} f d x, \quad \forall v \in S_{h}^{0} .
$$

Further combining (4.1) and (4.2) we get

$$
\bar{a}\left(u-u_{B}, v\right)+\sum_{e \in E_{h}^{\text {in }}} v\left(m_{e}\right) \int_{b_{e}} \sigma\left(u-u_{B}\left(m_{e}\right)\right) d x=0, \quad \forall v \in S_{h}^{0} .
$$

In the sequel we will first prove some lemmas that will be used in the analysis of problem (1.1). Next, denote by $\|\cdot\|_{1, \infty, K}$ the norm of $W^{1, \infty}(K)$, where $K \subset \mathbb{R}^{2}$, and if $B=\left(b_{i j}\right)_{i, j=1}^{2}$, let $\|B\|_{1, \infty, K}=\max _{1 \leq i, j \leq j}\left\|b_{i j}\right\|_{1, \infty, K}$.

Lemma 4.1: Let $B(x)=\left(b_{i j}(x)\right)_{i, j=1}^{2} \in G L\left(\mathbb{R}^{2}\right)$ for all $x \in \Omega$. Assume that $b_{i j} \in W^{1, \infty}(K)$ for every $K \in T_{h}, i, j=1,2$,

$$
\max _{x \in K}\|B(x)\|_{\mathbb{R}^{2}} \leq C h_{K} \quad \text { and } \quad\|B\|_{1, \infty, K} \leq C, \quad \forall K \in T_{h},
$$

with a constant $C$ independent of $K$. Then, there exists a positive constant $C_{1}$ such that

$$
\sum_{e \in E_{h}^{\text {in }}} v\left(m_{e}\right) \int_{\partial b_{e}}(B \nabla v) \cdot n d s \leq C_{1} h|v|_{1, h}^{2}, \quad \forall v \in S_{h}^{0}
$$

Proof: Using the Cauchy-Schwarz inequality, Lemma 3.5, Green's formula and the fact that $v$ is a linear polynomial in every $K \in T_{h}$, we estimate the left-hand side of (4.7) similarly as in (3.26). Applying then Lemma 3.6 we obtain the desired estimate (4.7). 
We introduce now in $S_{h}^{0}+H_{0}^{1}(\Omega)$ the norm $\|\cdot \mid\|\left\|_{h},\right\| v \|_{h}=\left(|v|_{1, h}^{2}+\|\sqrt{\sigma} v\|_{0, \Omega}^{2}\right)^{1 / 2}$.

Lemma 4.2: There exists a positive constant $C$ and $h_{0} \in(0,1)$, such that for all $h \in\left(0, h_{0}\right)$ and every $v \in S_{h}^{0}$, we have

$$
\bar{a}(v, v)+\sum_{e \in E_{h}^{\text {in }}} v^{2}\left(m_{e}\right) \int_{b_{e}} \sigma d x \geq C\|\| v \|_{h}^{2} .
$$

Proof: Since $A$ satisfies the coercivity condition (1.2), we have, for every $v \in S_{h}^{0}$,

$$
\alpha_{0}|v|_{1, K}^{2} \leq\left(A\left(z_{K}\right) \nabla v, \nabla v\right)_{K}, \quad \forall K \in T_{h},
$$

with $\alpha_{0}$ the constant in (1.2). Therefore, using (3.12) and the fact that $\operatorname{div}\left(A\left(z_{K}\right) \nabla v\right)=0$, for all $K \in T_{h}$ and $v \in S_{h}^{0}$, we have

$$
\begin{aligned}
& \alpha_{0}|v|_{1, h}^{2} \leq \sum_{K \in T_{h}}\left(A\left(z_{K}\right) \nabla v, \nabla v\right)_{K}=\sum_{e \in E_{h}^{\text {in }}} v\left(m_{e}\right) \sum_{K \in T_{h}\left(V_{e}\right)}\left(A\left(z_{K}\right) \nabla v, \nabla \varphi_{e}\right)_{K} \\
& =\sum_{e \in E_{h}^{\text {in }}} v\left(m_{e}\right) \sum_{K \in T_{h}\left(V_{e}\right)}\left\{\int_{\partial K}\left(A\left(z_{K}\right) \nabla v\right) \cdot n \varphi_{e} d s-\int_{K} \operatorname{div}\left(A\left(z_{K}\right) \nabla v\right) \varphi_{e} d x\right\} \\
& =\sum_{e \in E_{h}^{\text {in }}} v\left(m_{e}\right) \int_{e}\left(A\left(z_{K^{+e}}\right) \nabla v_{K^{+e}}-A\left(z_{K^{-e}}\right) \nabla v_{K^{-e}}\right) \cdot n_{e} d s, \quad \text { i.e., } \\
& \alpha_{0}|v|_{1, h}^{2} \leq-\sum_{e \in E_{h}^{\text {in }}} v\left(m_{e}\right)\left\{\int_{\partial b_{e}^{+} \backslash e}\left(A\left(z_{K^{+e}}\right) \nabla v\right) \cdot n d s+\int_{\partial b_{e}^{-} \backslash e}\left(A\left(z_{K^{-e}}\right) \nabla v\right) \cdot n d s\right\},
\end{aligned}
$$

with $n_{e}$ denoting the external normal vector of $K^{+e}$ to $e$. Using Taylor's theorem we easily see that assumptions (4.6) in Lemma 4.1 hold for $B(x):=A(x)-A\left(z_{K}\right), x \in K, K \in T_{h}$. Then, for $h$ small enough, according to (4.10) and Lemma 4.1, we have

$$
\bar{a}(v, v) \geq \alpha_{0}|v|_{1, h}^{2}-C_{1} h|v|_{1, h}^{2} \geq \alpha_{1}|v|_{1, h}^{2},
$$

with a constant $\alpha_{1}$ independent of $h$ and $C_{1}$ the constant in (4.7). Therefore,

$$
\bar{a}(v, v)+\sum_{e \in E_{h}^{\text {in }}} v^{2}\left(m_{e}\right) \int_{b_{e}} \sigma d x \geq c \mid\|v\|_{h}^{2}-\sum_{e \in E_{h}^{\text {in }}} \int_{b_{e}} \sigma\left(c v^{2}-v^{2}\left(m_{e}\right)\right) d x, \quad \forall v \in S_{h}^{0}
$$

with $c=\frac{1}{2} \min \left(1, \alpha_{1}\right)$ and $\alpha_{1}$ the constant in (4.11). Also, by an arithmetic geometric mean inequality, we have

$$
\sum_{e \in E_{h}^{\text {in }}} \int_{b_{e}} \sigma\left(c v^{2}-v^{2}\left(m_{e}\right)\right) d x \leq \bar{\sigma} \sum_{e \in E_{h}^{\text {in }}} \int_{b_{e}}\left(v-v\left(m_{e}\right)\right)^{2} d x \leq \bar{\sigma} h^{2}|v|_{1, h}^{2} \leq \bar{\sigma} h^{2}\|v \mid\|_{h}^{2} .
$$

Finally, combining (4.12) and (4.13) we obtain (4.8). 
Existence: Since solving (4.2) is equivalent to a linear system, in order to prove existence it suffices to prove uniqueness; this follows easily from Lemma 4.2.

The following two lemmas can be easily proved with simple calculations.

Lemma 4.3: Let $v \in \widehat{S}(\widehat{K})$; then

$$
\|v\|_{0, \widehat{K}}^{2}=\frac{1}{6} \sum_{i=1}^{3} v^{2}\left(\widehat{m}_{i}\right)
$$

Lemma 4.4: There exists a positive constants $C$, independent of $h$ and $K \in T_{h}$, such that,

$$
C\|v\|_{0, K}^{2} \leq \sum_{e \in E_{h}(K)} h_{e}^{2} v^{2}\left(m_{e}\right) \leq C^{-1}\|v\|_{0, K}^{2}, \quad K \in T_{h}, \forall v \in S_{h}^{0}
$$

where $h_{e}=|e|$.

Consider a triangle $K \in T_{h}$ and a side $e \in E_{h}^{\text {in }} \cap E_{h}(K)$. Let $n_{e}$ be the external normal vector of $K$ to $e$. According to section 2 we denote by $K^{+e}$ triangle $K$ and by $K^{-e}$ the other triangle of $T_{h}$, having $e$ as a common side with $K$. In analogy to (3.4), we introduce now the notation

$$
[A(x) \nabla \chi] \cdot n_{e}=\left(A(x) \nabla \chi_{K^{+e}}\right) \cdot n_{e}-\left(A(x) \nabla \chi_{K^{-e}}\right) \cdot n_{e}, \quad \forall x \in e, \forall \chi \in S_{h}^{0} .
$$

Lemma 4.5: Let $v, w \in S_{h}^{0}$. Then

$$
\begin{aligned}
a(v, w) & =\bar{a}(v, w)-\sum_{K \in T_{h}}(\operatorname{div}(A \nabla v), w-Q(w))_{K} \\
& +\sum_{K \in T_{h}} \int_{\partial K}(A \nabla v) \cdot n w d s-\sum_{e \in E_{h}^{\text {in }}} w\left(m_{e}\right) \int_{e}[A \nabla v] \cdot n_{e} d s,
\end{aligned}
$$

with $n_{e}$ as in (3.4) and $Q$ as in (3.39).

Proof: We apply Green's formula to $\int_{\partial b_{e}}(A \nabla v) \cdot n d s$ and $\int_{K} A \nabla v \cdot \nabla w d x$. Then the desired relation follows by simple calculations.

Now we will estimate the difference $u-u_{B}$ in the mesh dependent norm $\||\cdot|\|_{h}$.

Theorem 4.1: Let $u$ be the solution of (1.1) and $u_{B}$ the solution of (4.2). Then, for $h$ sufficiently small, there exists a constant $C$, independent of $h$, such that

$$
\left\|u-u_{B}\right\|_{h} \leq C\left(\sum_{K \in T_{h}} h_{K}^{2}\|u\|_{2, K}^{2}\right)^{1 / 2} .
$$

Proof: Using Lemma 4.2 and (4.5), and similar arguments as in (3.25), we have, for $\chi \in S_{h}^{0}$,

$$
\|\| u_{B}-\chi \mid \|_{h} \leq C \sup _{\substack{v \in S_{h}^{0} \\ v \neq 0}} \frac{\bar{a}(u-\chi, v)+\sum_{e \in E_{h}^{\text {in }}} v\left(m_{e}\right) \int_{b_{e}} \sigma\left(u-\chi\left(m_{e}\right)\right) d x}{\|v\|_{h}} .
$$


In view of $(2.1)$, it is obvious that $\left|b_{e}\right| \leq C \max \left(h_{K^{+e}}^{2}, h_{K^{-e}}^{2}\right) \leq C \varrho^{-2} h_{e}^{2}$, for any $e \in E_{h}^{\text {in }}$. Applying then Lemmas 4.4 and 3.7, we get

$$
\sum_{e \in E_{h}^{\text {in }}}\left|b_{e}\right| v^{2}\left(m_{e}\right) \leq C \sum_{K \in T_{h}} \sum_{e \in E_{h}(K)} h_{e}^{2} v^{2}\left(m_{e}\right) \leq C\|v\|_{0, \Omega}^{2} \leq C\|\| v \|_{h}^{2}, \quad \forall v \in S_{h}^{0}
$$

Using now the Cauchy-Schwarz inequality and (4.19), we obtain for all $v, \chi \in S_{h}^{0}$

$$
\sum_{e \in E_{h}^{\text {in }}} v\left(m_{e}\right) \int_{b_{e}} \sigma\left(u-\chi\left(m_{e}\right)\right) d x \leq C\|\| v\|\|_{h}\left(\sum_{e \in E_{h}^{\text {in }}} \int_{b_{e}} \sigma^{2}\left(u-\chi\left(m_{e}\right)\right)^{2} d x\right)^{1 / 2} .
$$

Then in view of Lemmas 3.5 and 3.6, Green's formula, the fact that $\chi$ is a linear polynomial in every $K \in T_{h}$ and that $a_{i j} \in C^{1}(\bar{\Omega}), i, j=1,2$, we have similarly as in (3.26) and (3.27)

$$
|\bar{a}(u-\chi, v)| \leq C\|\nabla v\|_{0, h} \sum_{K \in T_{h}}\left(h_{K}^{2}\|u\|_{2, K}^{2}+|u-\chi|_{1, K}^{2}\right), \quad \forall \chi, v \in S_{h}^{0}
$$

Choosing now $\chi=I u$ in (4.18), (4.20) and (4.21), we easily obtain

$$
\|\| u-u_{B} \mid \|_{h}^{2} \leq C\left(\sum_{K \in T_{h}} h_{K}^{2}\|u\|_{2, K}^{2}+\|u-I u\|_{1, h}^{2}+\sum_{e \in E_{h}^{\text {in }}} \int_{b_{e}} \sigma^{2}\left(u-I u\left(m_{e}\right)\right)^{2} d x\right) .
$$

Also, from a standard approximation property, cf., e.g., [7, Theorem 5], we get

$$
\sum_{e \in E_{h}^{\text {in }}} \int_{b_{e}} \sigma^{2}\left(u-I u\left(m_{e}\right)\right)^{2} d x \leq \bar{\sigma}^{2} \varrho^{-2} \sum_{K \in T_{h}} h_{K}^{2}|u|_{1, K}^{2}
$$

Finally, (4.22), (4.23) and (3.29) give (4.17).

Remark 4.1: In the case of the Galerkin/finite element method a similar $H^{1}$ norm error estimate holds.

Next, we will estimate the difference $u-u_{B}$ in the $L^{2}{ }^{2}$ norm.

Theorem 4.2: Let $u$ be the solution of (1.1) and $u_{B}$ the solution of (4.2). Assume that $f \in H^{1}(\Omega), a_{i j} \in C^{2}(\bar{\Omega}), 1 \leq i, j \leq 2, \sigma \in C^{1}(\bar{\Omega})$ and that the interior point $z_{K}$ is the barycenter of $K$, for every $K \in T_{h}$. Then, there exists a constant $C$, independent of $h$, such that

$$
\left\|u-u_{B}\right\|_{0, \Omega} \leq C h^{2}\left(\|u\|_{2, \Omega}+\|f\|_{1, \Omega}\right) .
$$

Proof: We consider the following auxiliary adjoint problem: Seek $\varphi \in H^{2}(\Omega)$ such that

$$
-\operatorname{div}\left(A^{T} \nabla \varphi\right)+\sigma \varphi=u-u_{B} \quad \text { in } \Omega \quad \text { and } \quad \varphi=0 \quad \text { on } \partial \Omega .
$$

It is well known that (4.25) has a unique solution and the following regularity estimate holds:

$$
\|\varphi\|_{2, \Omega} \leq C\left\|u-u_{B}\right\|_{0, \Omega}
$$


Using (4.25) and Green's formula, we easily obtain

$$
\begin{aligned}
\left\|u-u_{B}\right\|_{0, \Omega}^{2}= & a\left(u-u_{B}, \varphi-v\right)+\left(u-u_{B}, \sigma(\varphi-v)\right)_{\Omega}+a\left(u-u_{B}, v\right) \\
& +\left(u-u_{B}, \sigma v\right)_{\Omega}-\sum_{K \in T_{h}} \int_{\partial K}\left(u-u_{B}\right)\left(A^{T} \nabla \varphi\right) \cdot n d s, \quad \forall v \in S_{h}^{0} .
\end{aligned}
$$

Obviously,

$$
a\left(u-u_{B}, \varphi-v\right)+\left(\sigma\left(u-u_{B}\right), \varphi-v\right)_{\Omega} \leq C\left\|u-u_{B}\right\|_{1, h}\|\varphi-v\|_{1, h}, \quad \forall v \in S_{h}^{0} .
$$

Next, we will estimate the third and forth term of the right-hand side of (4.27). Using Lemma $4.5,(1.1)$ and Green's formula, we have for $v \in S_{h}^{0}$

$$
\begin{aligned}
& a\left(u-u_{B}, v\right)+\left(\sigma\left(u-u_{B}\right), v\right)_{\Omega}=\sum_{K \in T_{h}} \int_{\partial K}(A \nabla u) \cdot n v d s+\sum_{K \in T_{h}} \int_{K} f v d x-\bar{a}\left(u_{B}, v\right) \\
& -\left(\sigma u_{B}, v\right)_{\Omega}+\sum_{K \in T_{h}}\left(\operatorname{div}\left(A \nabla u_{B}\right), v-Q(v)\right)_{K}-\sum_{K \in T_{h}} \int_{\partial K}\left(A \nabla u_{B}\right) \cdot n v d s \\
& +\sum_{e \in E_{h}^{\text {in }}} v\left(m_{e}\right) \int_{e}\left[A \nabla u_{B}\right] \cdot n_{e} d s,
\end{aligned}
$$

with $Q$ as in (3.39). Applying similar arguments as in (3.40), we easily obtain

$$
\int_{e}[A \nabla u] \cdot n_{e} d s=0, \quad \forall e \in E_{h}^{\text {in }} .
$$

Also, in view of (4.30) and the fact that $v\left(m_{e}\right)=0$ for all $e \in E_{h} \backslash E_{h}^{\text {in }}$, we get

$$
\begin{aligned}
& \sum_{K \in T_{h}} \int_{\partial K}\left(A \nabla\left(u-u_{B}\right)\right) \cdot n v d s-\sum_{e \in E_{h}^{\text {in }}} v\left(m_{e}\right) \int_{e}\left[A \nabla\left(u-u_{B}\right)\right] \cdot n_{e} d s \\
& =\sum_{K \in T_{h}} \int_{\partial K}\left(A \nabla\left(u-u_{B}\right)\right) \cdot n\left(v-v\left(m_{e}\right)\right) d s, \quad \forall v \in S_{h}^{0} .
\end{aligned}
$$

Thus, using (4.31) and (4.4), in (4.29) we have

$$
\begin{aligned}
& a\left(u-u_{B}, v\right)+\left(\sigma\left(u-u_{B}\right), v\right)_{\Omega}=\sum_{K \in T_{h}} \int_{K} f(v-Q(v)) d x \\
& -\sum_{K \in T_{h}} \int_{K} \sigma\left(u_{B} v-Q\left(u_{B} v\right)\right) d x+\sum_{K \in T_{h}}\left(\operatorname{div}\left(A \nabla u_{B}\right), v-Q(v)\right)_{K} \\
& \quad+\sum_{K \in T_{h}} \int_{\partial K}\left(A \nabla\left(u-u_{B}\right) \cdot n\left(v-v\left(m_{e}\right)\right) d s, \quad \forall v \in S_{h}^{0},\right.
\end{aligned}
$$

In the sequel we will estimate the right-hand side of (4.32). In view of (3.45) and the fact that $C_{K}(f)$, defined in the proof of Theorem 3.2, is constant over $K$, we can rewrite the first two sums of (4.32) in the following way

$$
\int_{K} f(v-Q(v)) d x+\int_{K} \sigma\left(v u_{B}-Q\left(v u_{B}\right)\right) d x=\int_{K}\left(f-C_{K}(f)\right)(v-Q(v)) d x
$$




$$
+\int_{K}\left(\sigma-\sigma\left(z_{K}\right)\right)\left(u_{B} v-Q\left(u_{B} v\right)\right) d x, \quad \forall v \in S_{h}^{0}
$$

Thus, since $f \in H^{1}(K)$ and $\sigma \in C^{1}(\bar{\Omega})$, according to (3.43)-(3.47), we get

$$
\begin{aligned}
& \left|\int_{K} f(v-Q(v)) \sigma\left(v u_{B}-Q\left(v u_{B}\right)\right) d x\right| \leq C h_{K}^{2}\left(|f|_{1, K}|v|_{1, K}+\int_{K}\left|\nabla\left(u_{B} v\right)\right| d x\right) \\
& \leq C h_{K}^{2}\left(\|f\|_{1, K}+\left\|u-u_{B}\right\|_{1, K}\right)\left(\|\varphi\|_{1, K}+\|\varphi-v\|_{1, K}\right), \quad \forall v \in S_{h}^{0} .
\end{aligned}
$$

Next, we will estimate the third term on the right-hand side of (4.32). Also using (3.45), (3.46) and the fact that $a_{i j} \in C^{2}, 1 \leq i, j \leq 2$, we have for all $v \in S_{h}^{0}$, as above

$$
\sum_{K \in T_{h}}\left(\operatorname{div}\left(A \nabla u_{B}\right), v-Q(v)\right)_{K} \leq C h^{2}\left(\|u\|_{1, h}+\left\|u-u_{B}\right\|_{1, h}\right)\left(\|\varphi\|_{1, h}+\|\varphi-v\|_{1, h}\right) .
$$

In order to estimate the last term of (4.32) we add and subtract to it the term

$$
\sum_{K \in T_{h}} \sum_{e \in E_{h}(K)} A\left(m_{e}\right) \int_{e} \nabla\left(u-u_{B}\right) \cdot n\left(v-v\left(m_{e}\right)\right) d s .
$$

Using now Lemma 3.6, we obtain

$$
\begin{aligned}
& \left|\sum_{K \in T_{h}} \sum_{e \in E_{h}(K)} \int_{e}\left(A-A\left(m_{e}\right)\right) \nabla\left(u-u_{B}\right) \cdot n\left(v-v\left(m_{e}\right)\right) d s\right| \\
& \leq C h\left\|\nabla\left(u-u_{B}\right)\right\|_{0, h}|v|_{1, h}+C h^{2}\|u\|_{2, \Omega}|v|_{1, h}, \quad \forall v \in S_{h}^{0} .
\end{aligned}
$$

Next, in view of the fact that $v$ is a linear polynomial restricted on $e \in E_{h}$, and applying similar arguments as in (3.40) and (3.32), we get

$$
\begin{aligned}
& \sum_{K \in T_{h}} \sum_{e \in E_{h}(K)} A\left(m_{e}\right) \int_{e} \nabla\left(u-u_{B}\right) \cdot n\left(v-v\left(m_{e}\right)\right) d s \\
& =\sum_{K \in T_{h}} \sum_{e \in E_{h}(K)} A\left(m_{e}\right) \int_{e}\left(\nabla u \cdot n-\mathcal{M}_{e}^{0}(\nabla u \cdot n)\left(v-v\left(m_{e}\right)\right) d s\right. \\
& =\sum_{K \in T_{h}} \sum_{e \in E_{h}(K)} A\left(m_{e}\right) \int_{e}\left(\nabla u \cdot n-\mathcal{M}_{e}^{0}(\nabla u \cdot n)\left(v-v\left(m_{e}\right)-\varphi+\varphi\left(m_{e}\right)\right) d s\right. \\
& \leq C \sum_{K \in T_{h}} h_{K}\|u\|_{2, K}|v-\varphi|_{1, K} \leq C h\|u\|_{2, \Omega}\|v-\varphi\|_{1, h}, \quad \forall v \in S_{h}^{0} .
\end{aligned}
$$

Thus, combining (4.32)-(4.36), we obtain

$$
\begin{aligned}
& \left|a\left(u-u_{B}, v\right)+\left(u-u_{B}, \sigma v\right)_{\Omega}\right| \\
& \leq C h\left(\left\|u-u_{B}\right\|_{1, h}+h\left(\|u\|_{2, \Omega}+\|f\|_{1, \Omega}\right)\right)\left(\|\varphi\|_{1, h}+\|\varphi-v\|_{1, h}\right), \quad \forall v \in S_{h}^{0} .
\end{aligned}
$$

Finally, we estimate the last term of (4.27), as in (3.49). Thus, we get

$$
\left|\sum_{K \in T_{h}} \int_{\partial K}\left(A^{T} \nabla \varphi\right) \cdot n\left(u-u_{B}\right) d s\right| \leq C h|\varphi|_{2, \Omega}\left\|u-u_{B}\right\|_{1, h} .
$$


Therefore, choosing $v=I \varphi$ in (4.27) and using (4.28), (4.37), (4.38), (4.17), (4.26) and (3.29), we have

$$
\begin{aligned}
& \left\|u-u_{B}\right\|_{0, \Omega}^{2} \leq C\left\|u-u_{B}\right\|_{1, h}\|\varphi-I \varphi\|_{1, h}+C h\left(\left\|u-u_{B}\right\|_{1, h}\right. \\
& \left.\quad+h\left(\|u\|_{2, \Omega}+\|f\|_{1, \Omega}\right)\right)\left(\|\varphi\|_{1, \Omega}+\|\varphi-I \varphi\|_{1, h}\right) \leq C h^{2}\left(\|u\|_{2, \Omega}+\|f\|_{1, \Omega}\right)\left\|u-u_{B}\right\|_{0, \Omega}
\end{aligned}
$$

Remark 4.2: In the proof of Theorem 4.2, the hypotheses $f \in H^{1}(\Omega), a_{i j} \in C^{2}(\bar{\Omega}), 1 \leq i, j \leq 2$, $\sigma \in C^{1}(\bar{\Omega})$ are used in order to estimate the right-hand side of (4.29). In the case of a finite element method the corresponding $L^{2}$ error estimate holds without the term $\|f\|_{1, \Omega}$ in the right-hand side.

Acknowledgment. The author would like to thank Professor Charalambos Makridakis for suggesting the problem and Professors Charalambos Makridakis and Georgios Akrivis for contributing several valuable remarks. Also he would like to thank the referees for their comments that led to an improvement of the presentation.

\section{REFERENCES}

1. Bank, R.E., Rose, D.J., Some error estimates for the box method, SIAM J. Numer. Anal. 24 (1987), 777-787.

2. Brenner, S.C., Scott, L.R., The Mathematical Theory of Finite Element Methods, Springer-Verlag, New York, 1994.

3. Cai, Z., On the finite volume element method, Numer. Math. 58 (1991), 713-735.

4. Cai, Z., Mandel, J., McCormick, S., The finite volume element method for diffusion equations on general triangulations, SIAM J. Numer. Anal. 28 (1991), 392-402.

5. Chatzipantelidis, P., Finite Volume and Finite Element Methods for Boundary and Initial-Boundary Value Problems, PhD Thesis (Greek), University of Crete, Greece, 1998.

6. Ciarlet, P.G., Basic Error Estimates for Elliptic Problems, in Handbook of Numerical Analysis, Vol II, North-Holland, Amsterdam, 1991, pp. 17-351.

7. Ciarlet, P.G., Raviart, P.-A., General Lagrange and Hermite interpolation in $\mathbb{R}^{n}$ with applications to finite element methods, Arch. Rat. Mech. Anal. 46 (1972), 177-199.

8. Crouzeix, M., Raviart, P.-A., Conforming and nonconforming finite element methods for solving the stationary Stokes equation I, RAIRO-M² AN 7 (1973), 77-104.

9. Grisvard, P., Elliptic Problems in Nonsmooth Domains, Pitman, Massachusetts, 1985.

10. Ewing, R.E., Lazarov, R.D., Vassilevski, P.S., Local refinement techniques for elliptic problems on cellcentered grids I. Error analysis, Math. Comp. 56 (1991), 437-461.

11. Hackbusch, W., On first and second order box schemes, Computing 41 (1989), 277-296.

12. Lazarov, R.D., Mishev, I.D., Vassilevski, P.S., Finite volume methods for convection-diffusion problems, SIAM J. Numer. Anal. 33 (1996), 31-55.

13. Kossioris, G., Makridakis Ch., Souganidis, P.E., Finite volume schemes for Hamilton-Jacobi equations (Submitted).

14. Morton, K.W., Stynes, M., An analysis of the cell vetrex method, RAIRO-M² AN 28 (1994), 699-724.

15. Morton, K.W., Stynes, M., Süli, E., Analysis of a cell-vetrex finite volume method for convection-diffusion problems, Math. Comp. 66 (1997), 105-124.

16. Schmidt, T., Box schemes on quadrilateral meshes, Computing 51 (1994), 271-292.

17. Süli, E., The accurancy of cell vertex finite volume methods on quadrilateral meshes, Math. Comp. 59 (1992), 359-382.

18. Süli, E., Convergence of finite volume schemes for Poisson's equation on nonuniform meshes, SIAM J. Numer. Anal. 28 (1991), 1419-1430.

19. Temam, R., Navier-Stokes Equations, North-Holland, Amsterdam, 1979.

20. Vassilevski, P.S., Petrova S.I., Lazarov, R.D., Finite difference schemes on triangular cell-centered grids with local refinement, SIAM J. Sci. Stat. Comput. 13 (1992), 1287-1313.

21. Zlámal, M., On the finite element method, Numer. Math. 12 (1968), 394-409. 